\title{
Stressed deformable reflector design and pneumatic membrane antenna for cryogenic thermal vacuum chamber testing
}

\section{Marcos Esparza, Heejoo Choi, Hyukmo Kang, Christian d'Aubigny, Amarjiit Pandde, et al.}

Marcos Esparza, Heejoo Choi, Hyukmo Kang, Christian d'Aubigny, Amarjiit Pandde, Henry Quach, Aman Chandra, Karlene Karrfalt, Yuzuru Takashima, Art Palisoc, Jonathan W. Arenberg, Kristy Gogick Marshall, Christopher Glynn, Sean M. Godinez, Marcos Tafoya, Brandon Chalifoux, Christopher Walker, Daewook Kim, "Stressed deformable reflector design and pneumatic membrane antenna for cryogenic thermal vacuum chamber testing," Proc. SPIE 11820, Astronomical Optics: Design, Manufacture, and Test of Space and Ground Systems III, 118200R (24 August 2021); doi: 10.1117/12.2594403

Event: SPIE Optical Engineering + Applications, 2021, San Diego, California, United States 


\title{
Stressed Deformable Reflector Design and Pneumatic Membrane Antenna for Cryogenic Thermal Vacuum Chamber Testing
}

\author{
Marcos Esparza $^{\mathrm{a}, \Phi}$, Heejoo Choi ${ }^{\mathrm{a}, \mathrm{b}}$, Hyukmo Kang ${ }^{\mathrm{a}}$, Christian d'Aubigny ${ }^{\mathrm{c}}$, Amarjiit Pandde, Henry \\ Quach $^{\mathrm{a}}$, Aman Chandra ${ }^{\mathrm{d}}$, Karlene Karfalt ${ }^{\mathrm{a}}$, Yuzuru Takashima ${ }^{\mathrm{a}}$, Art Palisoc ${ }^{\mathrm{e}}$, Jonathan W. \\ Arenberg ${ }^{f}$, Kristy Gogick Marshall ${ }^{f}$, Christopher S. Glynn ${ }^{f}$, Sean M Godinez ${ }^{\mathrm{f}}$, Marcos Tafoya ${ }^{\mathrm{f}}$, \\ Brandon Chalifoux ${ }^{\mathrm{a}}$, Christopher Walker ${ }^{\mathrm{d}, \Psi}$, Daewook Kim ${ }^{\mathrm{a}, \mathrm{b}, \mathrm{d}, \Lambda}$ \\ ${ }^{a}$ Wyant College of Optical Sciences, Univ. of Arizona, 1630 E. Univ. Blvd., Tucson, AZ, 85721, \\ USA \\ ${ }^{b}$ Large Binocular Telescope Observatory, Univ. of Arizona 933 N Cherry Avenue, Tucson, AZ, \\ 85721, USA \\ ${ }^{c}$ Lunar and Planetary Laboratory, Univ. of Arizona, 1629 E Univ. Blvd, Tucson, AZ 85721 \\ ${ }^{\mathrm{d}}$ Department of Astronomy and Steward Observatory, Univ. of Arizona, 933 N. Cherry Ave., \\ Tucson, AZ, 85721, USA \\ éL'Garde, Inc., 15181 Woodlawn Avenue, Tustin, CA, 92780, USA \\ ${ }^{\mathrm{f}}$ Northrop Grumman Aerospace Systems, 1 Space Park Drive, Redondo Beach, CA, 90278, USA
}

\begin{abstract}
Deformable reflector technology has mainly been used for observations at visible and infrared wavelengths but has yet to be utilized for terahertz wavefront correction. We present an actuator for deformable reflectors that overcomes challenges particular to this wavelength such as a millimeter-scale stroke requirement. Bending moment actuators are used in both the radial and tangential directions to correct low-order wavefront aberrations. Strong and flexible materials such as Delrin are used for the reflector material. Such a deformable antenna can be used to correct wavefronts on future large radio antennae such as the Orbiting Astronomical Satellite for Investigating Stellar Systems (OASIS). This antenna uses a 20-meter thin membrane as its primary radio wave collector. A deformable reflector may be added to this system to allow for looser tolerances on the primary antenna shape and correct for wavefront errors inherent in an inflatable optic. To predict the wavefront errors that may be expected when using this type of thin membrane primary reflector, TVAC (Thermal Vacuum Chamber) test methods are also presented in these proceedings.
\end{abstract}

Keywords: radio astronomy, optomechanical design, deformable antenna, TVAC, membrane antenna, bending moment actuator

\section{INTRODUCTION}

The development of new radio antennae design concepts for stellar observation such as the Orbiting Astronomical Satellite for Investigating Stellar Systems (OASIS) require the advancement of supporting technologies such as deformable reflectors. OASIS is a proposed space observatory that will perform observations at terahertz frequencies and consists of a 20 -meter thin membrane primary reflector. ${ }^{1}$ Typical deformable mirrors for astronomical observation operate at visible and infrared wavelengths and have dynamic ranges on the order of 10 microns and nanometer repeatability. For a radio antenna operating at millimeter-scale wavelengths, a deformable reflector will need a dynamic range on the millimeter scale and precision on the order of 50 microns. We've developed, prototyped, and tested an actuator that utilizes bending moment actuation rather than the typical piston-type actuation (see Fig. 1) to achieve the required dynamic range. The actuator takes inspiration from the stressed-lap polisher used in the University of Arizona Mirror Lab. ${ }^{2}$

Фmaesparza@email.arizona.edu, ${ }^{\Psi}$ cwalker@as.arizona.edu, ${ }^{\Lambda}$ dkim@optics.arizona.edu 

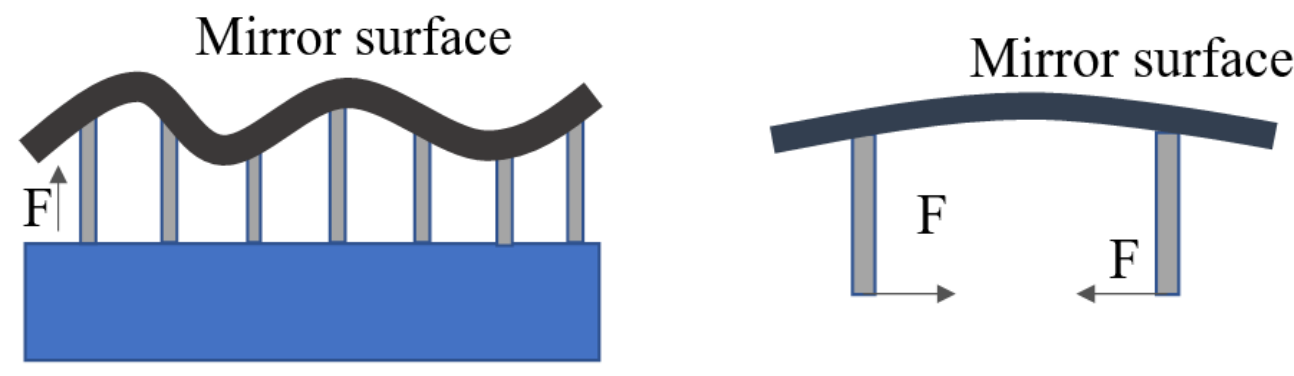

Figure 1. (left) A typical piston style deformable mirror and (right) a bending moment deformable reflector. The piston style deformable reflector applies a force normal to the reflector surface and the bending moment actuator applies for parallel to the reflector surface.

We also tested the surface figure on a 1-meter diameter mylar reflector prototype in a TVAC chamber to better understand the behavior of such an antenna in a space-like environment. These tests measuring the surface figure in multiple temperatures and pressures, with different inflation gases and after puncturing the reflector membrane.

\section{BENDING MOMENT-BASED DEFORMABLE REFLECTOR}

\subsection{Design of a Bending Moment Actuator Prototype for Deformable Reflectors}

A large-scale prototype of a bending moment actuator was designed, constructed, and tested to determine the feasibility of using such an actuator for a deformable reflector in a terahertz antenna. It was designed for large stroke $(5 \mathrm{~mm}$ of maximum deformation) and moderate repeatability ( 25 micron). The actuator was designed to bend a $12 \mathrm{~mm}$ thick Delrin plate. The plate is $100 \mathrm{~mm}$ wide and $425 \mathrm{~mm}$ long but the length of interest (what we are simulating as the "clear aperture") is $280 \mathrm{~mm}$ long. In the prototype, a stepper motor drives a leadscrew to translate a carriage. This carriage is attached to a lever arm which pulls on the turnbuckle and applies the bending moment. The turnbuckle is used to provide a static moment. These multiple stages allow for a large load to be applied at the turnbuckle with just a small stepper motor.
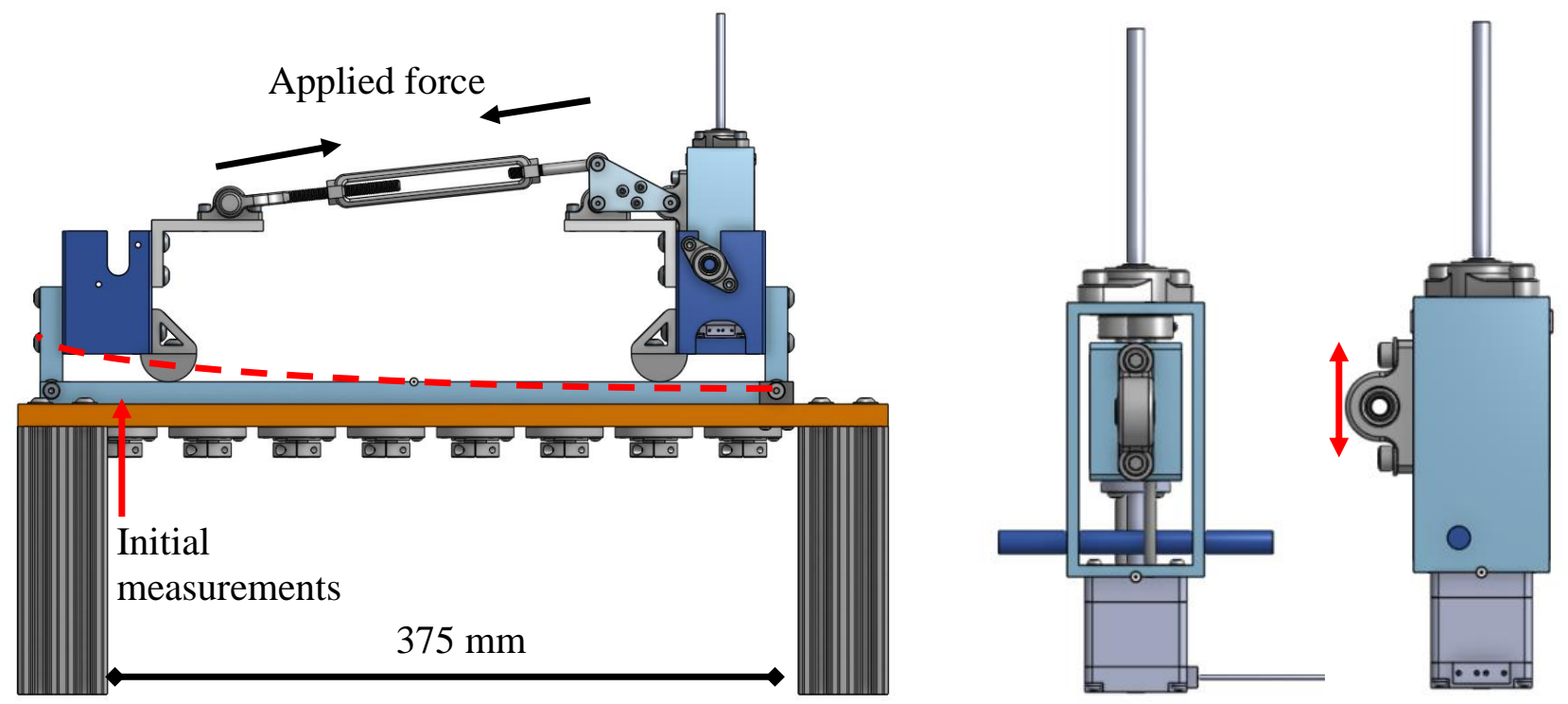

Figure 2. (left) CAD models of the bending moment actuator. The red, dashed curve shows the predicted deformation of the plate. The red arrow indicates the point where the initial displacement measurements were taken. Results of these measurements are shown in Fig. 4. (right) A CAD model of only the carriage and motor mount. The red arrow shows the direction of motion of the carriage. 

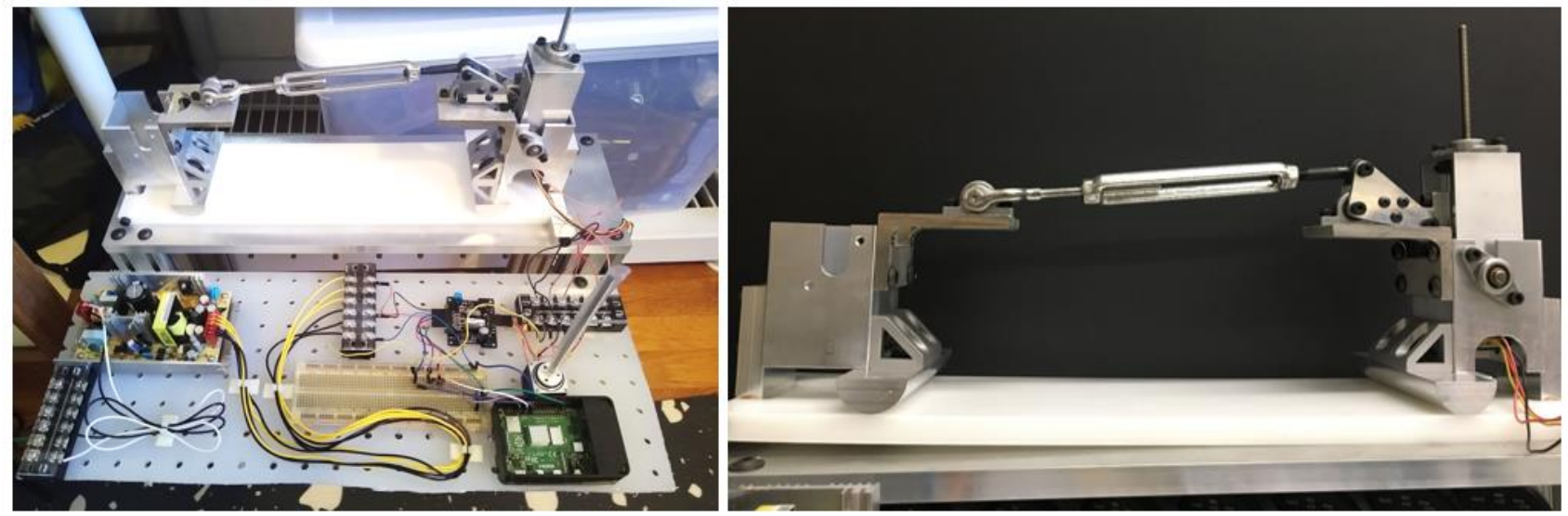

Figure 3. (left) Top view of the actuator prototype showing both the power and control electronics. (right) A front view of the actuator while it is deforming the Delrin plate.

\subsection{Testing of the Bending Moment Actuator Prototype for Deformable Reflectors}

To test the actuator, the plate-actuator assembly was fixed to an aluminum plate using a hinge on one side to constrain the location of a single side of the Delrin plate and actuator. The deformation of the plate was measured with eight digital dial indicators along the length of the plate, each with a resolution of 10 microns. For our initial tests, the displacement at the indicator furthest from the hinge constraint $(375 \mathrm{~mm})$ was measured. To test the repeatability of the actuator, the plate was deformed between two positions twenty times (Lower Pos and Upper Pos in Table 1.). This procedure was done at three different mean displacements which were $1.45 \mathrm{~mm}, 3.45 \mathrm{~mm}$, and $5.78 \mathrm{~mm}$. This corresponded to sending commands to the stepper motor to drive 500,1000, and 1500 steps respectively. Table 1 shows the results of these tests. While the repeatability (measured here as RMS) is within our tolerance (all but the upper position on $5.78 \mathrm{~mm}$ is less than 10 microns), there does seem to be drift in the system as shown in Table 1 and future design iterations of the prototype will aim to reduce that systematic error. Initial investigation into this systematic error will include adding a rotary encoder to the leadscrew as the drift error is likely being caused by the stepper motor skipping steps.

Table 1. Repeatability test results of the bending moment actuator. "Starting position" shows measurement from the dial indicator on the first trial and "End position" shows the measurement of the dial indicator on the twentieth trial. The drift was calculated by subtracting the end position from the starting position.

\begin{tabular}{lcccccc}
\hline Mean displacement & \multicolumn{2}{c}{$\mathbf{1 . 4 5} \mathrm{mm}$} & \multicolumn{2}{c}{$\mathbf{3 . 4 5} \mathrm{mm}$} & \multicolumn{2}{c}{$\mathbf{5 . 7 8 ~ m m}$} \\
& $\underline{\text { Lower Pos }}$ & $\underline{\text { Upper Pos }}$ & $\underline{\text { Lower Pos }}$ & $\underline{\text { Upper Pos }}$ & $\underline{\text { Lower Pos }}$ & $\underline{\text { Upper Pos }}$ \\
\hline Start position $(\mu \mathrm{m})$ & 100 & 1560 & 100 & 3650 & 100 & 5900 \\
End position $(\mu \mathrm{m})$ & 80 & 1520 & 90 & 3630 & 80 & 5840 \\
RMS $(\mu \mathrm{m})$ & 5.5 & 6.9 & 4.4 & 8.7 & 6.7 & 19 \\
Drift $(\mu \mathrm{m})$ & 20 & 40 & 10 & 20 & 20 & 60 \\
\hline
\end{tabular}

Future actuators are being developed. These have a smaller footprint to reduce the effects of the mount points on the deformable reflector surface. A simpler actuator concept (see Fig. 4) is currently being developed that consists of an array of actuators on a Delrin plate. Each actuator is made up of a linear actuator mounted on two posts. The posts are small load cells to allow for feedback control of the actuation. Analytical methods to predict the shape of the deformable reflector with multiple actuators with a particular actuator pattern are also being developed. 


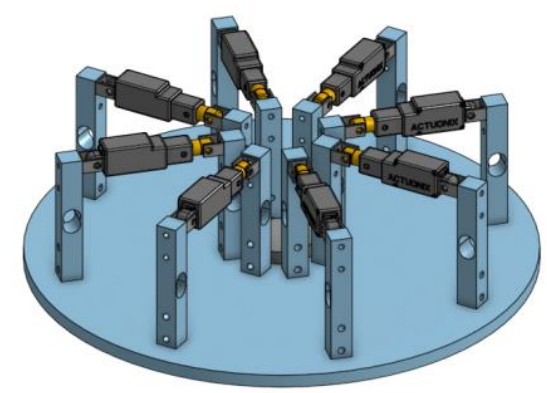

Figure 4. A CAD model of a deformable reflector design concept using bending moment actuators. The design utilizes linear actuators mounted on posts that are load cells to allow for force feedback.

\section{TESTING MEMBRANE REFLECTOR IN THERMAL VACUUM CHAMBER}

\subsection{Optomechanical design of the mounting system}

A large optical mount was designed and built to test a 1-meter inflatable membrane antenna (UUT, unit under test) in a TVAC at Northrop Grumman. The mount was designed to interface with an 8020 style carriage provided by Northrop Grumman. The 8020 carriage slid on linear rails and bearings and this provided the ability to load the UUT into the TVAC and adjust its position along the metrology optical axis. To adjust the rotation of the UUT about the $\mathrm{x}$-axis (see Fig. 5 for axes definition), two custom adjustment mechanisms was made (see Fig. 6 (left \& center)), one for each side of the UUT. These adjustment mechanisms were each mounted on the two 8020 trusses (one on each side of the UUT, see Fig 6 (left)) and the UUT was then mounted directly to these adjustment mechanisms. The adjust mechanism was made up of two custom aluminum plates, each with two mounted sleeve bearings sized for $3 / 8$ " shafts. The mounted sleave bearings were self-aligning up to $5 \mathrm{deg}$. A 3/8" shoulder bolt was put through the mounted sleave bearings on each aluminum plate. Each adjustment mechanism now had two sets (one on top and one on bottom) of two mounted sleave bearings and one shoulder bolt (with a nut). A set screw was then put above the top set and below the bottom set to constrain the two plates against each other and adjust the rotation about the X-axis. This adjustment mechanism allowed for \pm 3 degrees of rotation which was enough to ensure the fringes from the illumination source on the deflectometer were captured by the camera.
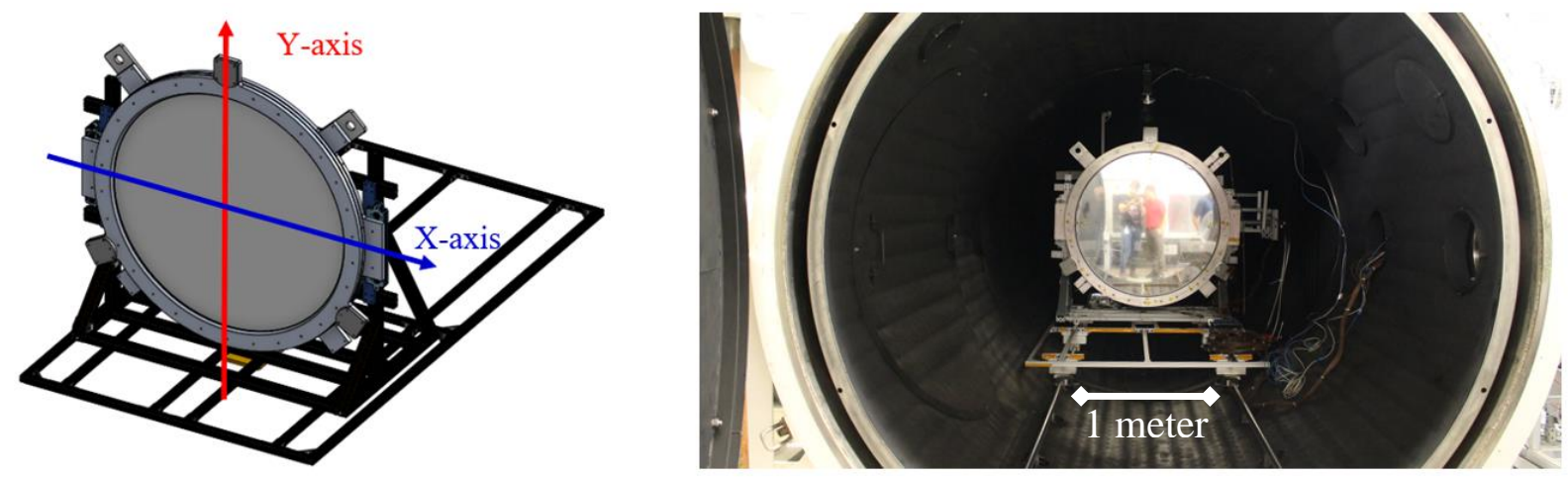

Figure 5. (left) A CAD model of the UUT on the custom optical mount with the axes labeled. (right) The UUT in the TVAC at Northrop Grumman

The trusses attached to the $\mathrm{X}$-axis rotation adjustment mechanism were connected by a center base. To adjust the rotation of the UUT about the Y-axis, another custom 1/2" aluminum plate was attached to the center base. A $3 / 4$ " shaft collar held a shaft that went through a mounted bearing attached to the slide carriage. This allowed for adjustments of the rotation about the Y-axis. The rotational angle could be adjusted by lightly tapping the mount at its outer most edge of the base with a small mallet and locking it down with a bracket. Translational adjustments about the $\mathrm{X}$ and $\mathrm{Y}$-axes were facilitated by the 8020 frame as it was fairly easy to loosen the 8020 brackets, shift the UUT to the desired position and 
tighten the bracket again. The adjustment sensitivity needed by the UUT mount was very large and easy to achieve due to the large dynamic range of the deflectometer.
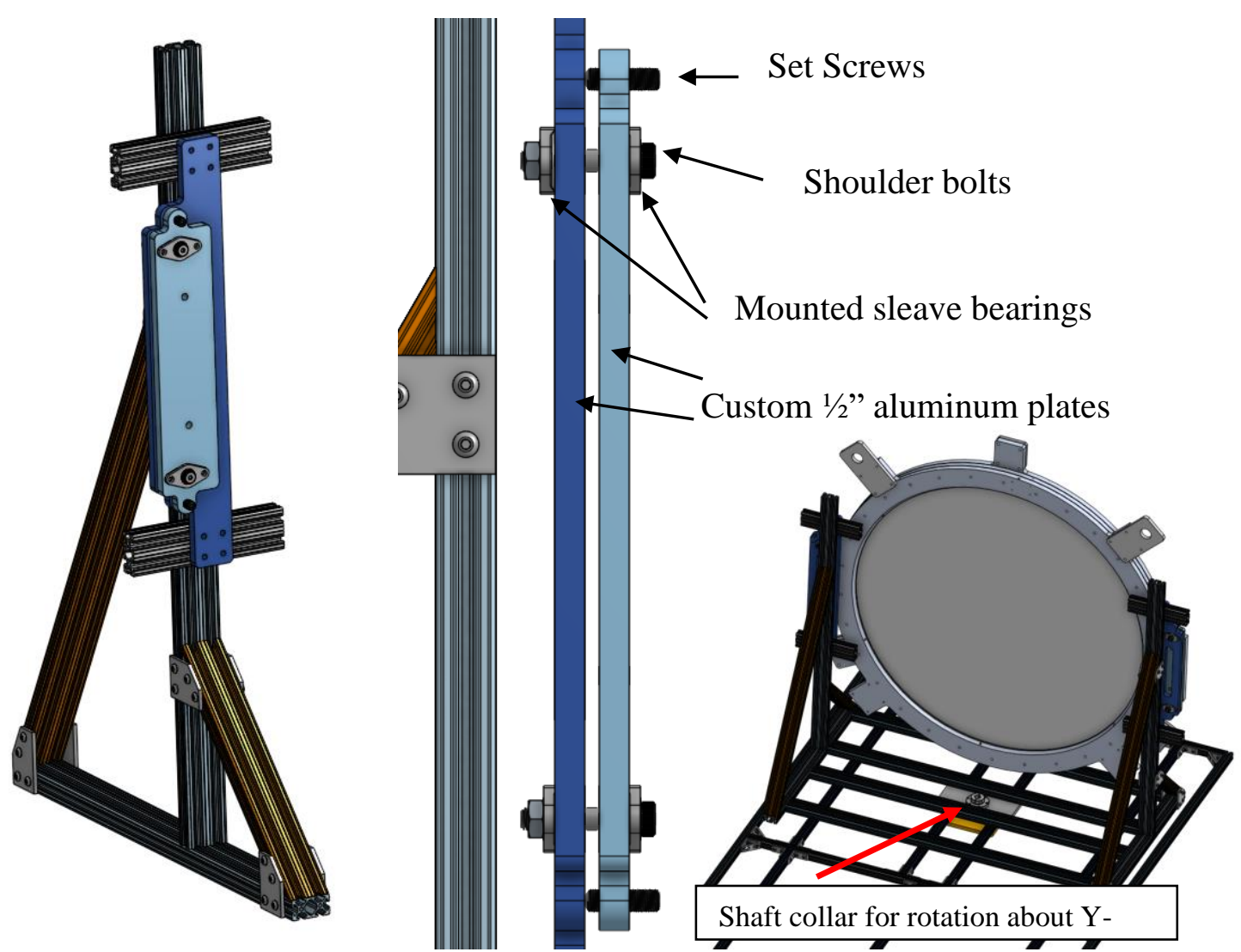

Figure 6. (left) A single truss used to mount the UUT, (center) the adjustment mechanism used to adjust tilt about the X-axis, (right) a CAD model of the UUT showing the point about which the UUT pivots for rotational adjustment about the Y-axis. This pivot point was meant to be at the vertex of the optic when it was inflated.

The pressure control system was placed in an enclosure with a heater so that it could be put in the TVAC chamber and be kept at a warmer operating temperature without the heat radiating out to the UUT (see Fig. 7 (left)). To inflate the membrane to a repeatable shape, three tooling balls were mounted behind the membrane (see Fig. 7 (right)). The reflector would be inflated until the influence of these tooling balls was observed and then the pressure was reduced by $10 \mathrm{~Pa}$ (the resolution of the pressure control system).
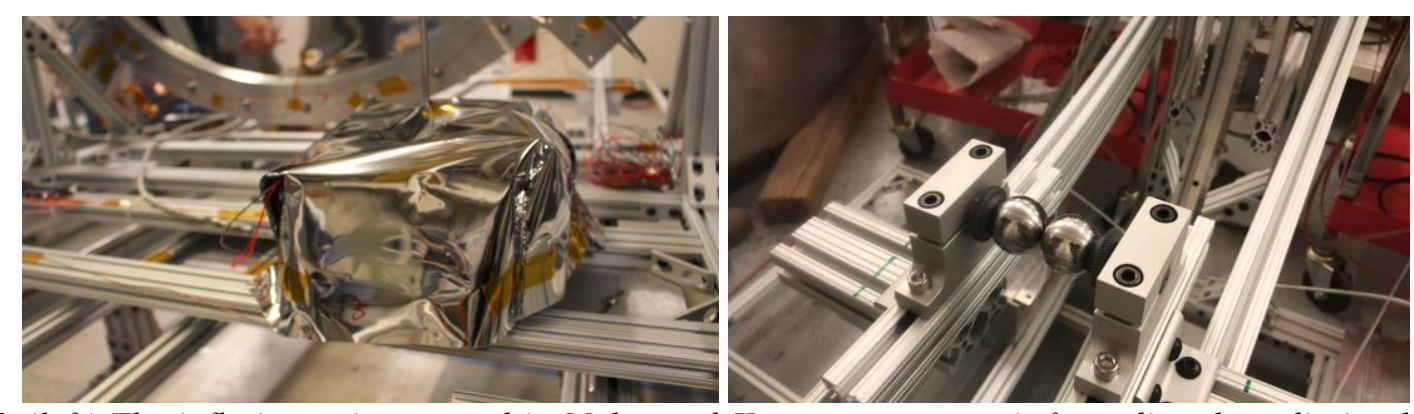

Figure 7. (left) The inflation unit wrapped in Mylar and Kapton to prevent it from directly radiating heat to the membrane and (right) One of three tooling balls that were used to ensure the membrane was inflated to a repeatable mechanical datum. 


\subsection{Deflectometry System for TVAC testing and Experimental setup at Northrop Grumman}

The deflectometer (see Fig 8. (left \& center)) used for this test was mounted on the 10-inch acrylic window at the end of the TVAC tank. The deflectometer consisted of a 12.9" iPad Pro (\#A2378) illumination source, a Point Grey monochrome camera (FL3-U3-13Y3M-C) with a $\mathrm{f}=12 \mathrm{~mm}$ lens, and a custom aluminum mount. The deflectometer was aligned once the UUT was placed in the TVAC tank and the chamber was closed. Spherical mounted retroreflectors were fixed to both the UUT and the deflectometer and a Leica laser tracker was used to measure the position of the UUT relative to the deflectometer. For more information about the deflectometer and the theory behind this metrology method, see the sister paper. ${ }^{3}$
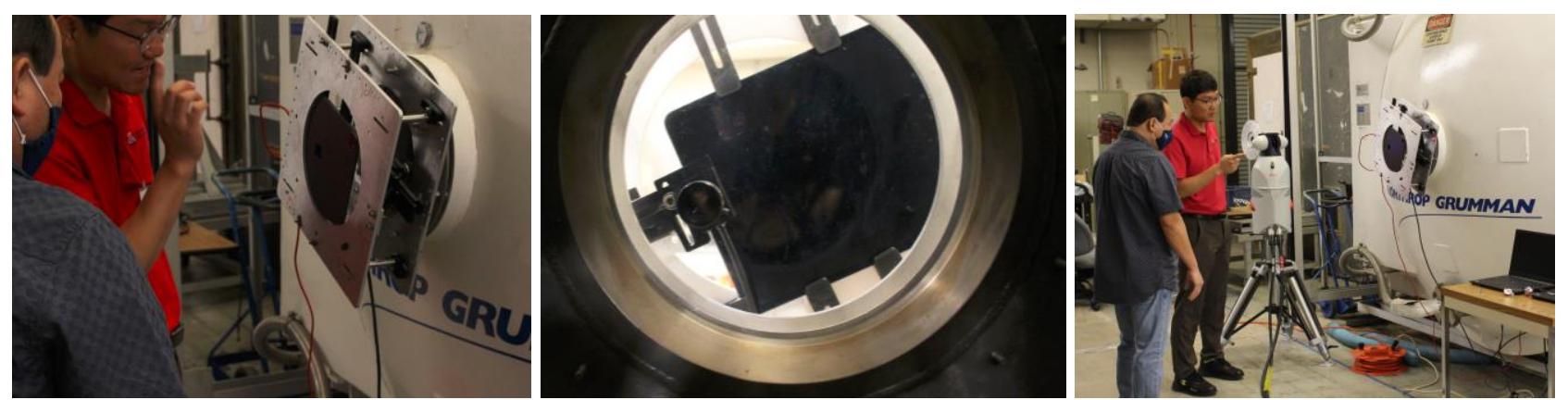

Figure 8. (left \& center) The deflectometer used to measure the surface figure.(right) The laser tracker used to measure the position of the UUT relative to the deflectometer.

The goal of the experiments done at Northrup Grumman was to gain a better understanding of how the membrane surface figure changed in different environments and conditions. These environments and conditions include testing in a laboratory environment $(\sim 300 \mathrm{~K}, \sim 100,000 \mathrm{~Pa})$, low temperature and low pressure (137 K, $0.11 \mathrm{~Pa})$, different gases inside the membrane (Helium, Argon and Xenon), an induced thermal gradient from a radiation source (Fig. 9 (left)), and puncturing the membrane from behind (see Fig. 9 (right) for photo of the puncture). Results and further details on this experimental setup can be found in the sister paper. ${ }^{4}$
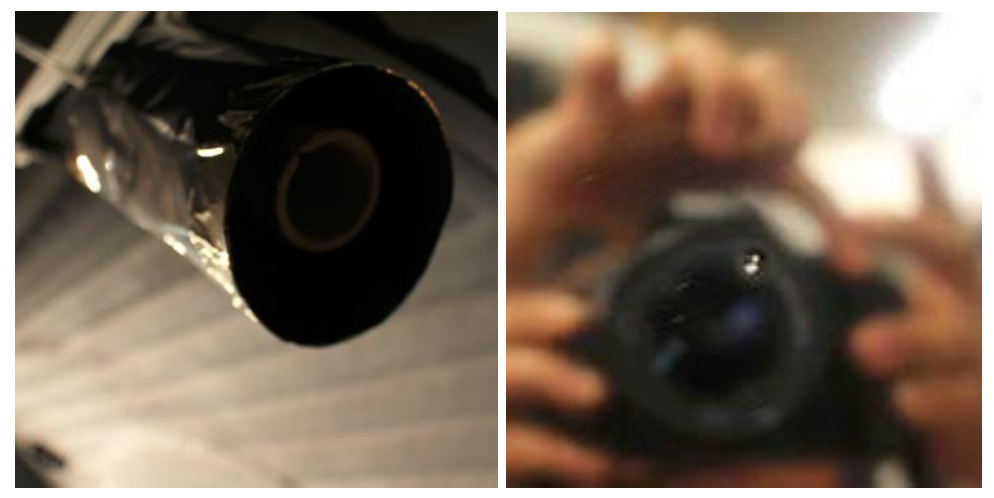

Figure 9. (left) The lamp used as a radiation source to induce a thermal gradient on the membrane, (right) the membrane after the puncture test.

\section{CONCLUSION AND FUTURE WORK}

With the development of bending moment actuators and TVAC testing methods for the 1-meter inflatable membrane prototype reflector (Fig. 10), technologies needed for observatories such as OASIS continue to be de-risked. Further development of bending moment actuators is being done to reduce the size and form factor and improve the repeatability of the actuators. Test results from the TVAC testing will continue to be analyzed and will influence the development of 
novel deformable reflectors as it informs what kinds of wavefront errors need to be corrected. Further metrology will also be done on the UUT to perform absolute measurements of the UUT surface figure.
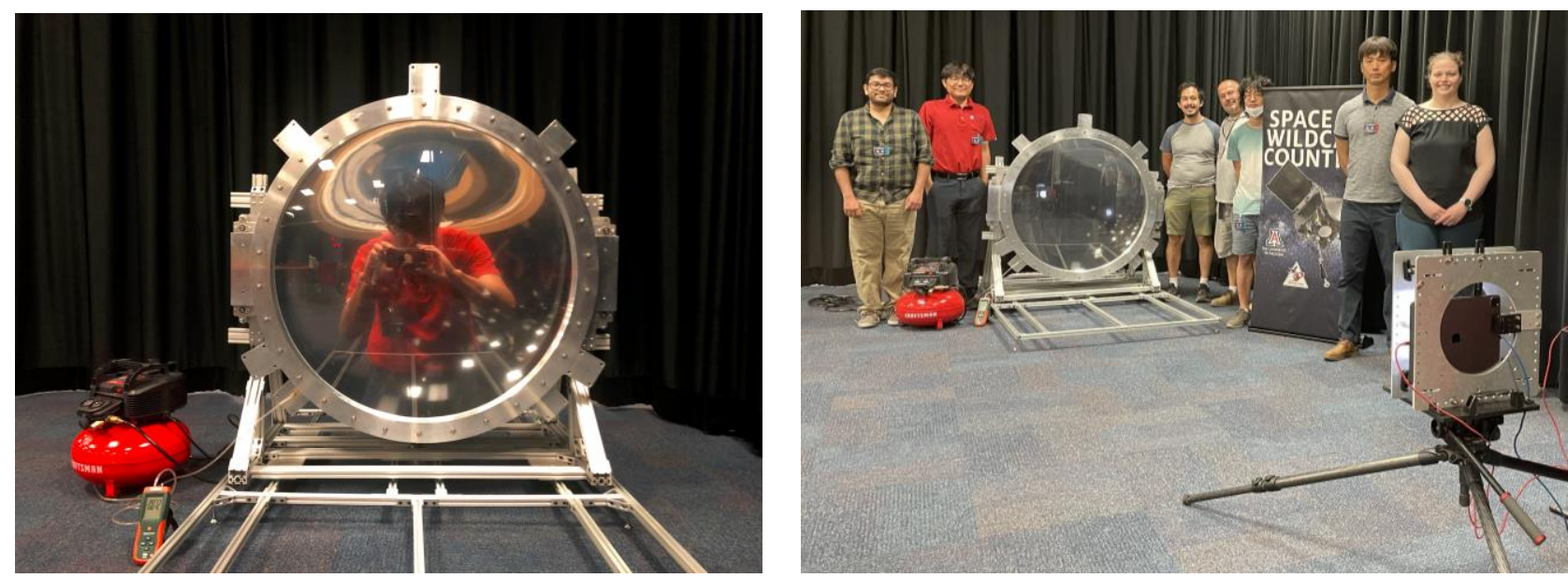

Figure 10. The UUT the optical mount and part of the team that helped build it at University of Arizona.

\section{ACKNOWLEDGEMENTS}

The authors would like to acknowledge the Friends of Tucson Optics Endowed Scholarships in Optical Sciences for helping support the design and metrology research done by the LOFT group. We would also like to thank FreeFall Aerospace in Tucson, Arizona for supplying the external inflation unit and providing help and expertise with its operation and wiring. We would lastly like to thank the team at Northrop Grumman Aerospace Systems for their work during the TVAC testing.

\section{REFERENCES}

[1] Walker, C., Kulesa, C., Smith, I. S., Perry, W., Kim, D., Palisoc, A., Cassapakis, C., Crowe, D. and Pierce, D., "Orbiting Astronomical Satellite for Investigating Stellar Systems (OASIS)" (2019).

[2] West, S. C., Martin, H. M., Nagel, R. H., Young, R. S., Davison, W. B., Trebisky, T. J., DeRigne, S. T. and Hille, B. B., "Practical design and performance of the stressed-lap polishing tool," Appl. Opt. 33(34), 8094-8100 (1994).

[3] Quach, H., Berkson, J., Sirsi, S., Choi, H., Dominguez, R., Duffy, B., Lesser, D., Takashima, Y., Palisoc, A., Walker, C. and Kim, D. W., "Full-aperture optical metrology for inflatable membrane mirrors," Proc.SPIE 11487 (2020).

[4] Quach, H., Esparza, M., Kang, H., Chandra, A., Choi, H., Berkson, J., Karrfalt, K., Sirsi, S., Takashima, Y., Palisoc, A., Arenberg, J. W., Marshall, K. G., Glynn, C. S., Godinez, S. M., Tafoya, M., Walker, C., D’Aubigny, C. D. and Kim, D., "Deflectometry-Based Thermal Vacuum Testing for a Pneumatic Terahertz Antenna," Astron. Opt. Des. Manuf. Test Sp. Gr. Syst. III, SPIE (2021). 